\title{
Platelet-derived growth factor promotes osteoblast proliferation by activating G-protein-coupled receptor kinase interactor-1
}

\author{
YOU WU, YING ZHANG, QINGSHUI YIN, HONG XIA and JIAJIA WANG \\ Department of Traumatic Orthopedics, General Hospital of Guangzhou Military Area Command of Chinese PLA, \\ Guangzhou, Guangdong 510010, P.R. China
}

Received September 5, 2013; Accepted March 19, 2014

DOI: $10.3892 / \mathrm{mmr} .2014 .2374$

\begin{abstract}
Platelet-derived growth factor (PDGF) has been reported to stimulate bone fracture-healing. Multiple studies have demonstrated that PDGF has a critical role in osteoblast or osteoprogenitor cell activation, although the underlying mechanism remains unclear. Studies have found that G-protein-coupled receptor kinase interactor-1 (GIT1) is activated by PDGF and described as an important factor in bone metabolism. In the present study, the effects of PDGF and GIT1 on the proliferation and apoptosis of osteoblasts were investigated in cultured osteoblasts isolated from rat calvaria with PDGF stimulation and GIT1 small interfering RNA transfection. The results demonstrated that PDGF rapidly stimulated GTI1 expression in osteoblasts, increased osteoblast proliferation and inhibited cell apoptosis. Furthermore, cyclin D1 expression was significantly upregulated, the number of cells in the G0/G1 phase was decreased, while the number in the $\mathrm{S}$ phase was increased. In cells with knockdown of GIT1, the change stimulated by PDGF was not evident. The results indicate that, PDGF stimulated GIT1 activation of cyclin D1 expression, thereby promoting osteoblasts to enter the $\mathrm{S}$ phase from the stationary $\mathrm{G} 0 / \mathrm{G} 1$ phase, leading to the proliferation of osteoblasts.
\end{abstract}

\section{Introduction}

Clinical studies have demonstrated that in patients with traumatic brain injury and fractures, excessive formation of callus or a variant of heterotopic ossification is commonly observed, which heals faster than fractures in patients with no brain injury (1). Therefore, it is possible that brain injury may be associated with accelerated fracture healing. However, the

Correspondence to: Dr Ying Zhang, Department of Traumatic Orthopedics, General Hospital of Guangzhou Military Area Command of Chinese PLA, 111 Liuhua Road, Guangzhou, Guangdong 510010, P.R. China

E-mail: zhangying_doc@yahoo.com.cn

Key words: platelet-derived growth factor, G-protein-coupled receptor kinase interactor-1, osteoblast, bone fracture-healing mechanism underlying this is unclear. Several studies have demonstrated that increased levels of circulating growth factors or cytokines stimulate the local release of growth factors, which in turn mediates increased osteogenesis (2). Platelet-derived growth factor (PDGF) is one of the most important growth factors and it has been demonstrated that ischemic insult and penetrating injury enhanced the expression of PDGF- $\beta(3,4)$.

The PDGF gene was identified to be expressed by numerous cell types during fracture healing in the human body, and appears to function to promote bone formation and reconstruction (5). As an important mitogen, PDGF is highly expressed in bone tissue trauma, and has an important role in the proliferation and differentiation of osteoblasts. PDGF promotes DNA synthesis in bone cells in early cell cycle stages, that stimulate the osteoblast entering from the static state G0/G1 to replicative S phase, increase secretory phase cells, enhance monocyte and fibroblast migration and induce local fibroblast proliferation and differentiation, thereby promoting bone formation (6). PDGF also promotes fibroblast growth in vitro and has a mitogenic effect on cells that originated from mesenchymal tissues, including osteoblasts, which facilitate bone formation and stimulate bone resorption (7). Therefore, PDGF is an important factor in accelerating fracture healing in traumatic brain injury and fracture.

The specific mechanism of PDGF in promoting fracture healing remains unclear. Previously, Ren et al (8) found that phosphorylation of the G-protein-coupled receptor kinase interacting protein 1 (GIT1) tyrosine 321 is required for focal adhesions and for PDGF-activated migration of osteoblast association with FAK. GIT1 widely exists in mammals and has an important role in cell growth and migration (9). This demonstrates that PDGF is able to promote osteoblast migration through activating GIT1; however, there have been no studies, to the best of our knowledge, investigating the proliferation and apoptosis of osteoblasts. Therefore, the present study aimed to determine the effect of PDGF and GIT1 on osteoblast proliferation and apoptosis.

\section{Materials and methods}

Cell culture and transfection. Primary osteoblastic cells were isolated from newborn CD1 mice (Institute of Laboratory 
Animal Sciences, Chinese Academy of Medical Sciences and Peking Union Medical College, Beijing, China) calvaria by digestion with $0.1 \%$ collagenase type IA and $0.2 \%$ dispase as previously described (10). The cells were cultured in Dulbecco's modified Eagle's medium (Invitrogen Life Technologies, Carlsbad, CA, USA) with $10 \%$ fetal bovine serum (FBS; Invitrogen Life Technologies), $100 \mathrm{U} / 1$ penicillin and $100 \mu \mathrm{g} / \mathrm{l}$ streptomycin, in a humidified atmosphere with $5 \% \mathrm{CO}_{2}$ at $37^{\circ} \mathrm{C}$. The cells were passaged four times and used in the experiments. For cell transfection, $1 \times 10^{5}$ cells were seeded in a 6-well plate. GIT1 small interfering (si)RNA (specific for rat GIT1;AAGCTGCCAAGAAGAAGCTAC) and negative control siRNA (AATTCTCCGACACGTGTCACT) were designed as previously described (11) and synthesized by Ambion Life Technologies (Guangzhou, Guangdong, China). GIT1 siRNA was prepared and transfected at $100 \mathrm{nM}$ for $24 \mathrm{~h}$ as previously described (12). Osteoblastic cells were serum-deprived for $24 \mathrm{~h}$ prior to the experimental treatments, then stimulated for $24 \mathrm{~h}$ with PDGF $(10 \mathrm{ng} / \mathrm{ml}$; Sigma-Aldrich, St. Louis, MO, USA).

Western blot. The cultured cells were washed with phosphate-buffered saline (PBS) and lysed on ice for 20 min in RIPA buffer. Next, the lysates were centrifuged at $10,000 \mathrm{x} \mathrm{g}$ for $10 \mathrm{~min}$ at $4^{\circ} \mathrm{C}$. The supernatants were collected and frozen at $-80^{\circ} \mathrm{C}$ or used immediately. The protein concentrations were determined by a bicinchoninic acid protein assay (Pierce Biotechnology, Inc., Rockford, IL, USA). A total of $40 \mu \mathrm{g}$ protein of each sample was heated for $10 \mathrm{~min}$ at $100^{\circ} \mathrm{C}$. Next, the samples were analyzed by $12 \%$ SDS-polyacrylamide gel electrophoresis (SDS-PAGE) and electroblotted onto nitrocellulose membranes. The membranes were blocked in 5\% non-fat milk for $1 \mathrm{~h}$ and then incubated with GIT1 antibody (a mouse monoclonal IgG1; Santa Cruz Biotechnology, Inc., Santa Cruz, CA, USA) overnight at $4^{\circ} \mathrm{C}$. GIT1 antibody-treated membranes were washed and incubated in the appropriate secondary antibody (1:7,000; Santa Cruz Biotechnology, Inc.) at $37^{\circ} \mathrm{C}$ for $1 \mathrm{~h}$. Following this, immune complexes were detected using the enhanced chemiluminescence kit (AppliChem, Darmstadt, Germany) and immunoreactive proteins were visualized by the Odyssey infrared imaging system (LI-COR Biosciences, Lincoln, NE, USA). The values were corrected with the absorbency of the internal control (GAPDH).

Cell proliferation analysis. The osteoblastic proliferation was measured by performing a 5-ethynyl-20-deoxyuridine (EdU) incorporation assay using an EdU Apollo DNA in vitro kit (Guangzhou RiboBio Co., Ltd., Guangzhou, China) according to the manufacturer's instructions. EdU $(100 \mu \mathrm{l})$ was added to the cultured cells and the cells were cultured for an additional $4 \mathrm{~h}$ at $37^{\circ} \mathrm{C}$. The cells were fixed with $4 \%$ formaldehyde for $15 \mathrm{~min}$ and treated with $0.5 \%$ Triton X-100 for $20 \mathrm{~min}$ at room temperature for permeabilization. Following washing with PBS three times, $100 \mu \mathrm{l}$ of 1X ApolloH reaction cocktail (Guangzhou RiboBio Co., Ltd.) was added to each well and the cells were incubated for $30 \mathrm{~min}$ at room temperature. Next, the cells were stained with $100 \mu \mathrm{l}$ Hoechst 33342 for $30 \mathrm{~min}$ and visualized under a fluorescent microscope (Olympus Corporation, Tokyo,

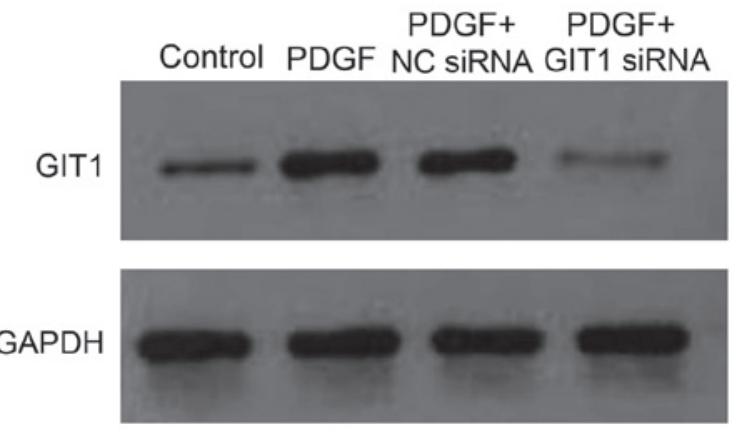

Figure 1. Expression of GIT1 in osteoblasts. The osteoblast cells were transfected with GIT1 siRNA or NC siRNA, and stimulated with or without PDGF. GIT1, G-protein-coupled receptor kinase interactor-1; siRNA, small interfering RNA; GIT1-siRNA, GIT1-specific siRNA; PDGF, platelet-derived growth factor; NC, negative control.

Japan). The EdU positive cells were counted using Image-Pro Plus 6.0 software (Media Cybernetics, Bethesda, MD, USA). All experiments were conducted in triplicate and three independent experiments were performed.

Flow cytometric analysis of the cell cycle. Cell cycle analysis was performed by flow cytometry. Briefly, the cultured cells were trypsinized into single cell suspensions and fixed with $70 \%$ ethanol for $30 \mathrm{~min}$ on ice. The cells were then stained with propidium iodide (Sigma-Aldrich). The labeled cells were washed with PBS and then analyzed by FACS Calibur flow cytometry (Becton-Dickinson, Franklin Lakes, NJ, USA) equipped with the ModiFit LT v2.0 software (Phoenix Flow Systems, San Diego, CA, USA). The proportions of cells in $\mathrm{G0} / \mathrm{G} 1$ and $\mathrm{S}$ phases were represented as DNA histograms. For each experiment, 10,000 events/sample were recorded.

Cell apoptosis analysis. For apoptosis analysis, terminal deoxynucleotidyl transferase-mediated dUTP nick end labeling (TUNEL) assay was performed on the cultured cells. The cells from each sample were processed using the In situ cell Death Detection kit (Roche Diagnostics, Hong Kong, China) according to the manufacturer's instructions. The adherent cells were grown on the slides, fixed with $4 \%$ formaldehyde for $25 \mathrm{~min}$ at $4^{\circ} \mathrm{C}$ and treated with $0.2 \%$ Triton X-100 for $5 \mathrm{~min}$ at room temperature for permeabilization. The cells were equilibrated in the equilibration buffer for $10 \mathrm{~min}$, then $50 \mu \mathrm{l}$ TDT buffer was added and incubated at $37^{\circ} \mathrm{C}$ for $1 \mathrm{~h}$ in a humidified chamber. The cells were washed with $2 \mathrm{X}$ SSC three times for $10 \mathrm{~min}$ each and incubated with $0.3 \% \mathrm{H}_{2} \mathrm{O}_{2}$ at room temperature for $10 \mathrm{~min}$. Streptavidin was then used to incubate the cells at room temperature for $30 \mathrm{~min}$, stained with $0.5 \mu \mathrm{g} / \mathrm{ml}$ DAPI for $5 \mathrm{~min}$ at room temperature in the dark and visualized under a fluorescent microscope (Olympus Corporation).

Statistical Analysis. All of the experiments were repeated three times. The results are expressed as the mean \pm standard deviation. The data were analyzed using SPSS 17.0 software (SPSS, Inc., Chicago, IL, USA). Comparisons between groups were analyzed using Student's t-test. $\mathrm{P}<0.05$ was considered to indicate a statistically significant difference. 


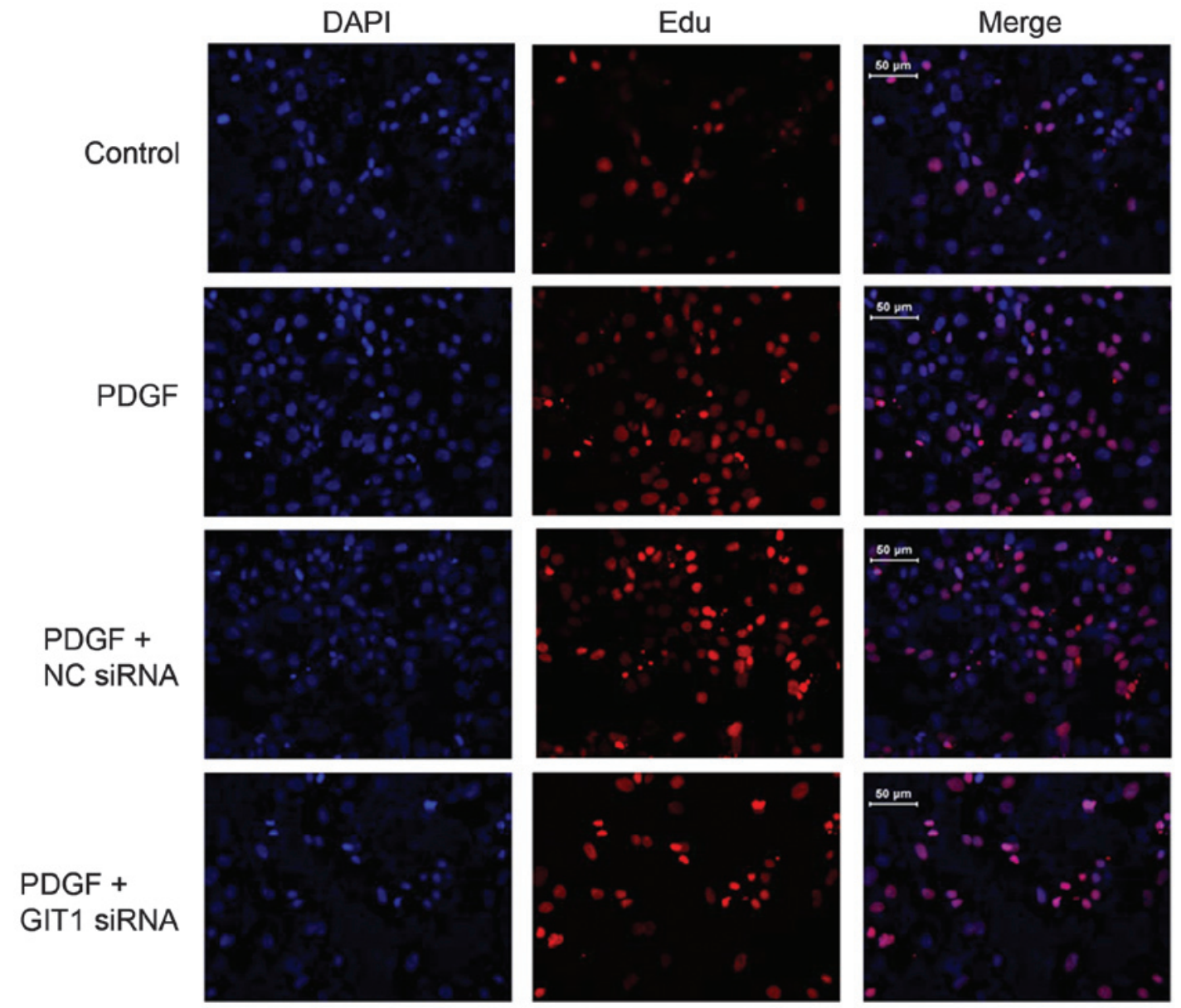

Figure 2. Effects of PDFG and GIT1 on the proliferation of osteoblasts. The osteoblastic proliferation was measured by performing a EdU incorporation assay using an EdU Apollo DNA in vitro kit. The data represent three independent experiments. The results of EdU incorporation assay were analyzed by confocal microscopy (scale bar, $50 \mu \mathrm{M}$ ) in osteoblasts infected with GIT1 siRNA or NC siRNA, and stimulated with or without PDGF. GIT1, G-protein-coupled receptor kinase interactor-1; PDGF, platelet-derived growth factor; NC, negative control; siRNA, small interfering RNA.

\section{Results}

PDGF stimulates GIT1 expression in osteoblasts. To determine the effects of PDGF on GIT1 expression, GIT1 expression in rat osteoblasts in response to PDGF was examined. Western blot analysis demonstrated that GIT1 expression significantly increased in osteoblasts following $24 \mathrm{~h}$ stimulation with PDGF compared with the non-PDGF group (control group; Fig. 1). In addition, GIT1-specific siRNA (GIT1-siRNA) was designed and transferred into osteoblasts. In the cells transfected with GIT1-siRNA, there was no significant difference in the GIT1 expression in the PDGF-stimulated cells compared with the control group.

GIT1 is required for PDGF-stimulated osteoblast proliferation. It is suggested that PDGF is an important cytokine for fracture healing. To determine the function of PDGF, PDGF was added in cultured osteoblasts from rats and the growth rate of osteoblasts was examined using the EdU incorporation assay. The growth rate of osteoblasts was significantly increased following stimulation with PDGF compared with the control group (Fig. 2). The results suggested that PDGF promoted osteoblast proliferation. As GIT1 expression was significantly increased in osteoblasts following PDGF stimulation, it was hypothesized that PDGF-mediated osteoblast proliferation occurs via GIT1 activation. To provide evidence for the function of GIT1 in osteoblast proliferation, the expression of GIT1 in osteoblasts was examined using specific siRNAs. The results demonstrated that cells transfected with GIT1-siRNA significantly reduced the osteoblasts growth induced by PDGF, which suggested that GIT1 is critical for PDFG-induced osteoblast proliferation. However, the cells transfected with GIT1-siRNA that were stimulated with PDGF proliferated faster than those in the control group, which implied other downstream targets may be involved in PDGF-stimulated osteoblast proliferation.

Effects of PDGF and GIT1 on osteoblasts cell cycle. To investigate the mechanism of PDGF on the proliferation of osteoblasts, the stages of the cell cycle that were regulated by PDGF in osteoblasts were examined. As expected, PDGF markedly increased the proportion of cells in $\mathrm{S}$ phase, with a corresponding decrease in the number of cells in the G0/G1 phase (Fig. 3) compared with the control group, while PDGF-induced cells transfected with GIT1-siRNA had no significant change in cell cycle stage. These results indicate that the knockdown of GIT1 inhibited osteoblast transition from the stationary phase (G0/G1 phase) to the replicative 


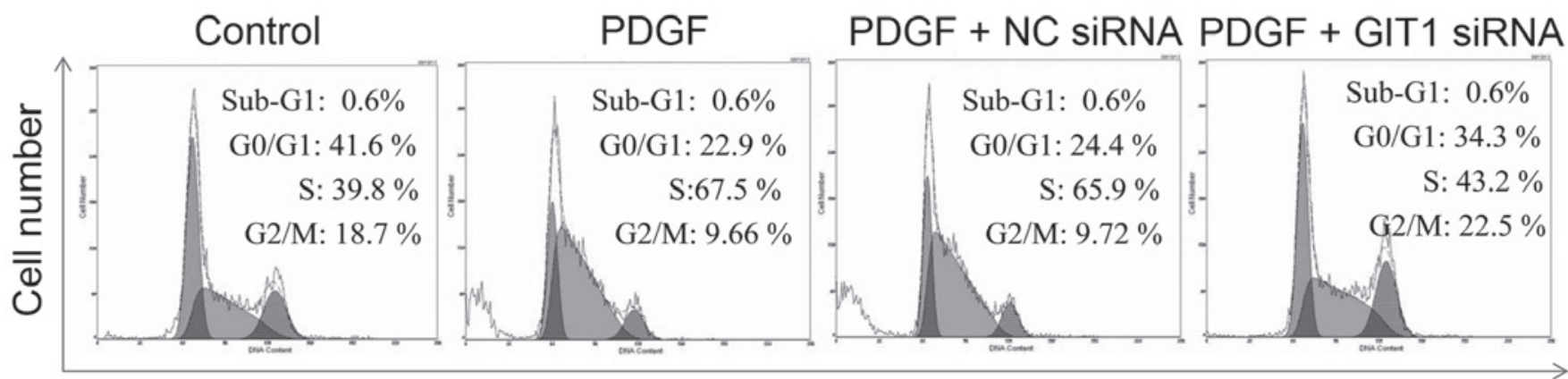

\section{DNA content}

Figure 3. The effects of PDFG and GIT1 on cell cycle distribution. The cells were seeded into 6-well plates and incubated for 24 h, and then GIT1 siRNA or NC siRNA transfection was conducted using Lipofectamine 2000. Following stimulation with or without PDGF for another $24 \mathrm{~h}$, the cell cycle distribution was analyzed by flow cytometry. GIT1, G-protein-coupled receptor kinase interactor-1; siRNA, small interfering RNA; GIT1-siRNA, GIT1-specific siRNA; PDGF, platelet-derived growth factor; NC, negative control.

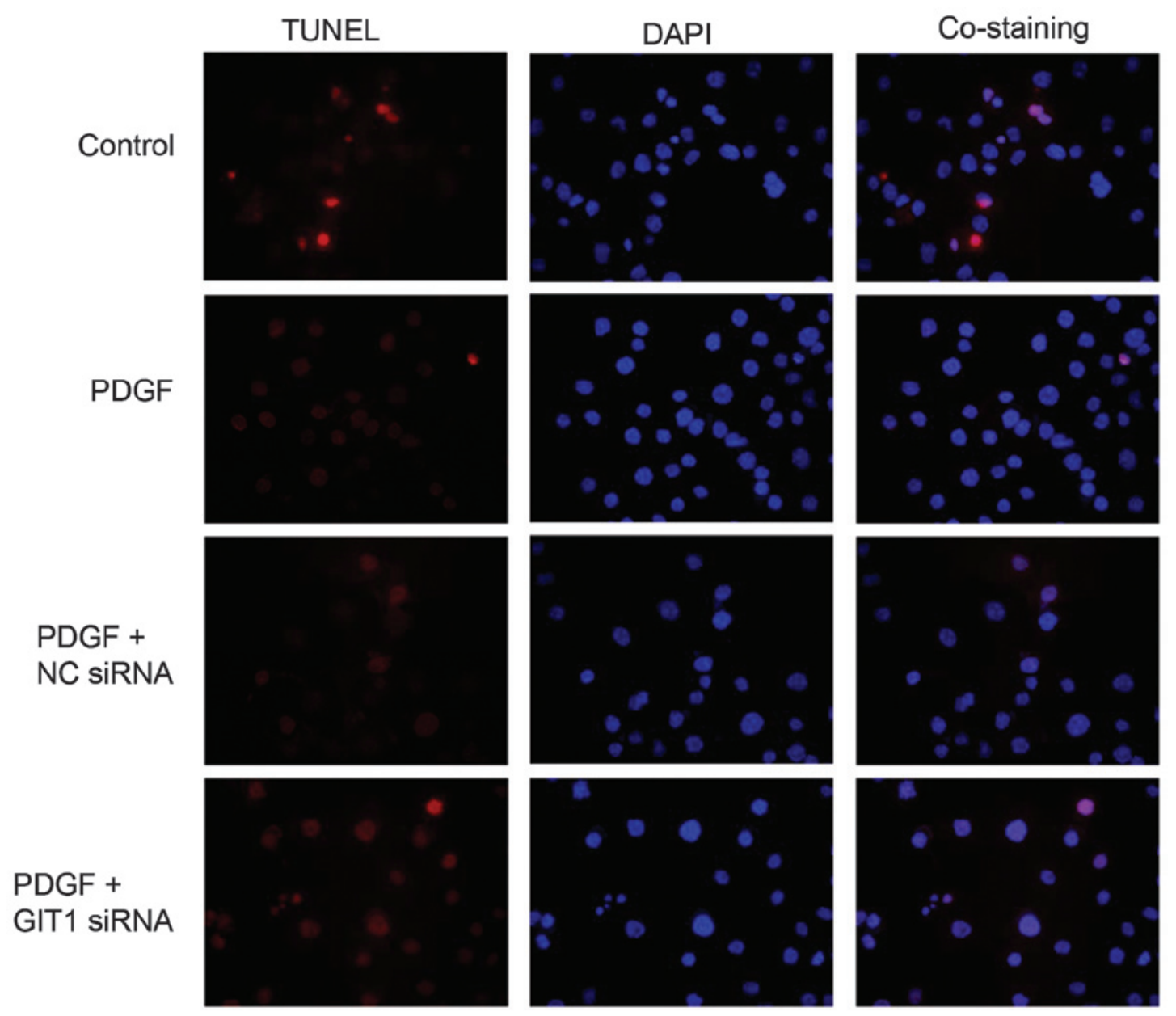

Figure 4. GIT1 on the anti-apoptotic effects of PDGF on osteoblasts. The cells were seeded into 6-well plates and incubated for $24 \mathrm{~h}$, and then transfected with GIT1 siRNA or NC siRNA. Following this, $10 \mathrm{ng} / \mathrm{ml}$ PDGF was then added to stimulate the osteoblasts for $24 \mathrm{~h}$. The cell apoptosis was determined by TUNEL-DAPI co-staining assay. GIT1, G-protein-coupled receptor kinase interactor-1; siRNA, small interfering RNA; PDGF, platelet-derived growth factor; GIT1-siRNA, GIT1-specific siRNA; NC, negative control; TUNEL, terminal deoxynucleotidyl transferase dUTP nick end labeling.

phase (S phase). Therefore, PDGF promotes osteoblast proliferation possibly through the activation of GIT1.

PDGF inhibits osteoblast apoptosis through upregulating GITI expression. To elucidate the role of PDGF and GIT1 in osteoblast apoptosis, a TUNEL assay was utilized to examine the effect of PDGF for osteoblast apoptosis. PDGF-induced osteoblast apoptosis significantly decreased compared with the non-PDGF cells (Fig. 4). These data demonstrated that PDGF was able to inhibit osteoblast apoptosis. No significant decrease in apoptosis was identified in the cells transfected with GIT1-siRNA when stimulated by PDGF. These results suggest that knockdown of 
GIT1 expression inhibited the anti-apoptotic function of PDGF in osteoblasts.

\section{Discussion}

PDGF is secreted by platelets during the early phases of fracture-healing and has been identified to have pivotal roles in the fracture healing process. Andrew et al (5) and Horner et al (7) demonstrated the widespread expression of PDGF-A chain and PDGF- $\alpha$ receptor in human bone formation, using in situ hybridization and immunohistochemistry, and indicated that this growth factor may exert autocrine and paracrine effects to regulate osteogenesis during skeletal development. PDGF was able to stimulate osteoblast and chondrocyte proliferation, promoting cartilage formation and intramembranous ossification in callus, the mRNA expression of which was highest in callus in the late stages of fracture (11). Therefore, it is important to investigate the specific mechanism of action of PDGF on osteoblast proliferation and understand the molecular mechanisms involved in fracture healing.

In the present study, it was demonstrated that GIT1 expression was significantly increased in osteoblasts following stimulation with PDGF, suggesting that PDGF was able to promote GIT1 expression. GIT1 is a multidomain scaffold protein expressed in various cell types, including neurons, endothelial cells and vascular smooth muscle cells. GIT1 is rapidly emerging as a scaffolding protein that has multiple binding domains for several different molecules, including ARF, Rac1, Cdc42, GTPases, PAK, PIX, MEK1 and PLC (12). In this manner, GIT1, through its function as an adaptor protein, is able to regulate protrusive activity and cell migration (13). A study demonstrated that GIT1 was expressed in osteoblasts and osteoclasts (14), and may participate in bone metabolism. GIT1 knockdown in mice resulted in increased bone mass due to disorganized podosome belt formation in osteoclasts resulting in impaired bone resorption, by affecting RANK signaling pathways (15). It has been demonstrated that the GIT1Y321F mutation inhibited PDGF-induced osteoblastic cell migration. Phosphorylation of tyrosine 321 of GIT1 is required for PDGF-induced association with FAK activation in focal adhesions for osteoblastic cell migration (8). The aforementioned studies demonstrate that the mechanism underlying GIT protein regulation of bone metabolism involves adjusting the dynamic changes of the cytoskeleton and acting as a scaffold protein involved in signal transduction.

In the present study, the effect of PDGF on rat primary osteoblasts was examined and it was demonstrated that PDGF induced a marked increase in cell growth and a decrease in the apoptosis of osteoblasts. Lee et al (15) revealed that the PDGF-BB releasing molded PLLA-TCP (porous poly (L-lactide)-tricalcium phosphate) membrane was demonstrated to be effective for continuous stimulation of bone regeneration when injected into rabbits. PDGF-BB may induce bone regeneration at early stages, in combination with a space making PLLA-TCP guide membrane. Knockdown of GIT1 expression resulted in no evident changes in the cell proliferation and apoptosis following PDGF stimulation, indicating that knockdown of GIT1 inhibited the cell proliferation of PDGF stimulated osteoblasts. This is consistent with an earlier study, which demonstrated that GIT1 may promote the cell proliferation and inhibit cell apoptosis (16). Thus, the results implicated that
PDGF may efficiently promote the cell proliferation of osteoblasts through activating GIT1. However, the cells transfected with GIT1-siRNA that were stimulated with PDGF grew faster than the control group, which implied other downstream targets were involved in PDGF-stimulated osteoblasts proliferation as well as GIT1.

An analysis of the cell cycle distribution in PDGF-treated osteoblasts using flow cytometry revealed that PDGF treatment increased the number of cells in the $S$ phase and decreased the cell numbers in the G0/G1 phase. This indicated that PDGF promoted cell entry into the S phase in osteoblasts and that the cyclin D1 protein level was increased following treatment with PDGF. However, effects of PDGF on the cell cycle and the cyclin D1 protein level exhibited no significant difference compared with that of the cells transfected with GIT1-siRNA. Yang et al (6) demonstrated that in osteoblasts pretreated by PDGF-AA for 24 h, G0/G1 phase was decreased and the number of cells in S and G2-M phases increased. PDGF-A mRNA may be expressed by human fetal osteoblasts and the level of its expression is enhanced by PDGF-AA and PDGF-BB. It is suggested PDGF-AA enhances cell replication by accelerating the cell cycle and inducing the quiescent cells into the proliferation phase of the cell cycle to modulate the fetal bone formation.

Cyclin D1, which belongs to the D group of cyclins, is expressed in all mammalian cells, except lymphocytes and myeloid cells. It varies in abundance with the cell cycle and peaks mid-G1 phase (17). In a number of cell types, cyclin D1 is rate-limiting for cell cycle progression and its abundance varies with the cell cycle $(18,19)$. In the present study it was demonstrated that activation of GIT1 was required for PDGF to upregulate cyclin D1 and stimulated cell cycle progression. A study demonstrated that GIT1 has an important role in cell proliferation and apoptosis, which is essential for maintaining normal postnatal mitochondrial integrity and cardiac function (20). GIT1 and GIT2, binds to the CTT (COOH-terminal tail) of IP3R and inhibits apoptosis by regulation of the IP3R-mediated $\mathrm{Ca}^{2+}$ signal (21). Furthermore, PDGF increased GIT1 tyrosine phosphorylation in osteoblasts and this response was observed for PDGF treatments up to $6 \mathrm{~h}$ after treatment and began to decline there after. GIT1 is the key phosphorylation site mediating PDGF-induced ERK1/2 activity, ERK1/2-GIT1 interactions and VEGF mRNA expression in osteoblasts (22). This implied that GIT1 may be a more potent downstream factor to mediate the PDGF proliferation effects in osteoblasts.

In conclusion, these findings extend the understanding of the crucial role of PDGF in osteoblast proliferation and provide insights into the novel regulatory relationship between PDGF and GIT1.

\section{References}

1. Huang W, Li Z, Li Z and Yang R: Does traumatic brain injury result in accelerated mandibular fracture healing? J Oral Maxillofac Surg 70: 2135-2142, 2012.

2. Onodera S, Nishihira J, Yamazaki M, Ishibashi T and Minami A: Increased expression of macrophage migration inhibitory factor during fracture healing in rats. Histochem Cell Biol 121: 209-217, 2004.

3. Iihara K, Sasahara M, Hashimoto N, Uemura Y, Kikuchi H and Hazama F: Ischemia induces the expression of the platelet-derived growth factor-B chain in neurons and brain macrophages in vivo. J Cereb Blood Flow Metab 14: 818-824, 1994. 
4. Takayama S, Sasahara M, Iihara K, Handa J and Hazama F: Platelet-derived growth factor B-chain-like immunoreactivity in injured rat brain. Brain Res 653: 131-140, 1994.

5. Andrew JG, Hoyland JA, Freemont AJ and Marsh DR Platelet-derived growth factor expression in normally healing human fractures. Bone 16: 455-460, 1995.

6. Yang D, Chen J, Jing Z and Jin D: Platelet-derived growth factor (PDGF)-AA: a self-imposed cytokine in the proliferation of human fetal osteoblasts. Cytokine 12: 1271-1274, 2000.

7. Horner A, Bord S, Kemp P, Grainger D and Compston JE: Distribution of platelet-derived growth factor (PDGF) A chain mRNA, protein, and PDGF-alpha receptor in rapidly forming human bone. Bone 19: 353-362, 1996.

8. Ren Y, Yu L, Fan J, et al: Phosphorylation of GIT1 tyrosine 321 is required for association with FAK at focal adhesions and for PDGF-activated migration of osteoblasts. Mol Cell Biochem 365: 109-118, 2012.

9. Manabe R, Kovalenko M, Webb DJ and Horwitz AR: GIT1 functions in a motile, multi-molecular signaling complex that regulates protrusive activity and cell migration. J Cell Sci 115: 1497-1510, 2002.

10. Inoue D, Santiago P, Horne WC and Baron R: Identification of an osteoclast transcription factor that binds to the human $\mathrm{T}$ cell leukemia virus type I-long terminal repeat enhancer element. J Biol Chem 272: 25386-25393, 1997.

11. Park YJ, Ku Y, Chung CP and Lee SJ: Controlled release of platelet-derived growth factor from porous poly(L-lactide) membranes for guided tissue regeneration. J Control Release 51: 201-211, 1998

12. Hsu RM, Tsai MH, Hsieh YJ, Lyu PC and Yu JS: Identification of MYO18A as a novel interacting partner of thePAK2/betaPIX/GIT1 complex and its potential function in modulating epithelial cell migration. Mol Biol Cell 21: 287-301, 2010.

13. Gavina M, Za L, Molteni R, Pardi R and de Curtis I: The GIT-PIX complexes regulate the chemotactic response of rat basophilic leukaemia cells. Biol Cell 102: 231-244, 2010.
14. Menon P, Yin G, Smolock EM,Zuscik MJ, Yan C and Berk BC: GPCR kinase 2 interacting protein 1 (GIT1) regulates osteoclast function and bone mass. J Cell Physiol 225: 777-785, 2010

15. Lee SJ, Park YJ, Park SN, et al: Molded porous poly (L-lactide) membranes for guided bone regeneration with enhanced effects by controlled growth factor release. J Biomed Mater Res 55: 295-303, 2001.

16. Pang J, Xu X, Wang X, et al: G-protein-coupled receptor kinase interacting protein-1 mediates intima formation by regulating vascular smooth muscle proliferation, apoptosis, and migration. Arterioscler Thromb Vasc Biol 33: 999-1005, 2013.

17. Baldin V, Lukas J, Marcote MJ, Pagano M and Draetta G: Cyclin D1 is a nuclear protein required for cell cycle progression in G1. Genes Dev 7: 812-821, 1993.

18. Xiong W, Pestell RG, Watanabe G, Li J, Rosner MR and Hershenson MB: Cyclin D1 is required for S phase traversal in bovine tracheal myocytes. Am J Physiol 272: L1205-L1210, 1997.

19. Beier F, Lee RJ, Taylor AC, Pestell RG and LuValle P. Identification of the cyclin D1 gene as a target of activating transcription factor 2 in chondrocytes. Proc Natl Acad Sci USA 96: 1433-1438, 1999

20. Pang J, Xu X, Getman MR, et al: G protein coupled receptor kinase 2 interacting protein 1 (GIT1) is a novel regulator of mitochondrial biogenesis in heart. J Mol Cell Cardiol 51: 769-776, 2011.

21. Zhang S, Hisatsune C, Matsu-Ura T and Mikoshiba K: G-protein-coupled receptor kinase-interacting proteins inhibit apoptosis by inositol 1,4,5-triphosphate receptor-mediated $\mathrm{Ca}^{2+}$ signal regulation. J Biol Chem 284: 29158-29169, 2009.

22. Rui Z, Li X, Fan J, et al: GIT1Y321 phosphorylation is required for ERK1/2- and PDGF-dependent VEGF secretion from osteoblasts to promote angiogenesis and bone healing. Int J Mol Med 30: 819-825, 2012. 